\title{
LA CARPETA DOCENTE. \\ INSTRUMENTO PARA LA EVALUACIÓN \\ DE LA CALIDAD DOCENTE
}

\author{
THE TEACHING PORTFOLIO. INSTRUMENT FOR TEACHING \\ QUALITY ASSESSMENT
}

O PORTFÓLIO DOCENTE. INSTRUMENTO PARA A AVALUAÇÃO DA
QUALIDADE DOCENTE

\author{
Anabel Galán-Mañas \\ Departament de Traducció i Interpretació i Estudis d'Àsia Oriental \\ Universitat Autònoma de Barcelona \\ ORCID: https://orcid.org/0000-0002-5498-9171 \\ isabel.galan@uab.cat
}

\begin{abstract}
Resumen: El objetivo de este artículo es presentar la carpeta docente como instrumento de evaluación del desempeńo del profesorado universitario. En primer lugar se indican las principales características de la carpeta y, posteriormente, se propone una estructura y se describen los elementos que podrían incluirse en cada uno de los apartados. Entre las principales conclusiones, se destaca que se trata de un documento que requiere un proceso de elaboración largo y complejo y que todavía está siendo infrautilizado en los procesos de contratación y acreditación. No obstante, sirve para la reflexión sobre la calidad y sobre el propio desempeño docente, fomenta el diseño de un plan de mejora y permite que el profesorado se autorregule, ya que al tratarse de un documento dinámico, que se debe revisar periódicamente, promueve la incorporación paulatina de mejoras. En definitiva, es un instrumento integrador, que recopila una diversidad de artefactos sobre la docencia procedentes de diferentes agentes que debería ser más valorado por las instituciones.
\end{abstract}

Palabras clave: Calidad docente. Carpeta docente. Evaluación de la docencia. Evaluación del desempeño docente. Portfolio docente.

Abstract: The aim of this article is to present the teaching portfolio as an instrument to assess university teachers' performance. Firstly, the main characteristics of the portfolio are outlined. Next, a structure is proposed, and the elements that could be included in each of the sections are described. Among the main conclusions, it stands out that this is a document that requires a long and complex elaboration process, and that it is still being underused in the hiring and certification processes. Nevertheless, it is useful to reflect on one's teaching quality and performance. It encourages the creation of an improvement 
plan and enables teachers' selfregulation, since, as it is a dynamic document that must be reviewed periodically, it stimulates the gradual incorporation of improvements. In short, it is an integrating instrument that compiles a diversity of artefacts on teaching from different agents that should be more highly valued by institutions.

KeYwords: Teaching dossier. Teaching assessment. Teaching performance assessment. Teaching portfolio. Teaching quality.

Resumo: O objetivo deste artigo é apresentar o portfólio docente como instrumento de avaliação do desempenho do professorado universitário. Em primeiro lugar, são indicadas as principais características do portfólio e, posteriormente, é proposta uma estrutura e são descritos os elementos que podem ser incluídos em cada uma das secçóes. Entre as principais conclusóes principais, destaca-se que se trata de um documento que requer um processo de elaboração longo e complexo e que ainda está sendo subutilizado nos processos de contratação e credenciamento. No entanto, ele serve para refletir sobre a qualidade e sobre o próprio desempenho do ensino, incentiva a concepção de um plano de melhora e permite ao professorado a autoregulamento, pois é um documento dinâmico, que deve ser revisto periodicamente, e promove a incorporação gradual de melhorias. Em suma, é um instrumento integrador que compila uma diversidade de artefato didático de diferentes agentes que deveria ser mais valorizado pelas instituiçóes.

Palavras-chave: Dossiê de ensino. Avaliação do ensino. Avaliação do desempenho docente. Portfólio docente. Qualidade do ensino.

\section{Introducción}

La Unión Europea considera que el futuro de Europa depende en cierto modo del trabajo colectivo y la mejora de la calidad de la educación. Un componente clave de ello es la calidad de la educación universitaria (Comisión Europea, 2018, p.6). De hecho, la calidad de la enseñanza es uno de los pilares del Espacio Europeo de Educación Superior.

La Asociación Europea para la Garantía de la Calidad de la Educación Superior sostiene que el profesorado es el recurso de aprendizaje más valioso al que acceden los estudiantes, y es el profesorado el garante de la calidad de la enseńanza. Para que esto sea posible, el profesorado debe tener total conocimiento de la materia que imparte, la competencia y la experiencia para transmitir su conocimiento de forma efectiva al estudiantado en contextos diferentes. La Asociación también apunta que su capacidad, compromiso, motivación y predisposición son factores clave.

No obstante, Fenstermacher and Richardson (2005) consideran que esos factores son insuficientes y que para alcanzar la calidad docente deben 
cumplirse no solo los criterios de buena enseñanza, sino también de enseñanza eficiente. Es decir, que no es suficiente que el profesorado utilice una metodología adecuada para enseńar, sino que el alumnado debe realmente devenir competente en lo que se le enseñe.

En este sentido, la Comisión Europea (2017) apunta que sin sistemas e instituciones de educación superior eficaces Europa no podrá responder a las necesidades del mercado, puesto que hacia el 2025 se prevé que la mitad de los puestos de trabajo requieran cualificaciones e alto nivel. Para analizar la eficacia de las instituciones de educación superior se pondrá en marcha una iniciativa para el seguimiento de los titulados para analizar cómo progresan en sus carreras profesionales (Comisión Europea 2017).

La mayoría de universidades en Europa utiliza un sistema de evaluación continua para evaluar la calidad docente de su profesorado. Con ello, intentan medir aspectos como la dedicación docente, la formación o la innovación. Entre los instrumentos para medir la calidad docente podemos encontrar: la observación en clase, el análisis de los materiales de enseñanza-aprendizaje, los informes de autoevaluación, los cuestionarios de evaluación de los alumnos y la carpeta docente.

El objetivo de este artículo es analizar la carpeta docente como instrumento para la evaluación de la docencia. En primer lugar, se define la calidad docente y se describen las competencias que debe dominar cualquier docente. Posteriormente, se describe la carpeta docente, sus usos y su contenido. Por último, se analiza de qué manera la carpeta docente permite evaluar de forma integrada las competencias docentes.

\section{La carpeta docente}

A pesar de que la carpeta docente (en adelante CD) es un instrumento aún poco conocido en algunos países, en Canadá y en el mundo anglosajón está muy extendido. Recibe diferentes denominaciones tanto en español (carpeta docente, portfolio docente, portafolios docente, portafolio educativo), como en inglés (teaching portfolio, teaching dossier).

Tiene su origen en el portafolio profesional de artistas y arquitectos (Little 2002). En el ámbito educativo se usa con diferentes fines: la carpeta de aprendizaje, para recoger muestras del desempeño del alumna- 
do (Paulson, Paulson, and Meyer (1991), Davies and Lemathieu (2003) y Driessen et al. (2005), Colén, Giné, Imbernón 2006, 50; Prendes and Mar 2010, Galán-Mañas 2009, 2016a, Galán-Mañas et al (2013).), la carpeta profesional, para recoger el desempeńo de la competencia profesional del alumnado (Galán-Mañas 2019), y la carpeta docente para recoger el desempeño del profesorado (Galán-Mañas et al 2013; Fuentes et al. 2011 a y b; Fernández March, 2004; Edgerton, R. et al. (1995).

Se trata de un instrumento en el que se describe y documenta el contenido y la eficacia de la propia docencia durante un período determinado mostrando el conjunto de competencias y méritos que posee y de qué modo el alumnado adquiere las competencias enseńadas.

Como apunta Fernández March (2004), la carpeta supone un giro metodológico en relación con los modelos anteriores de evaluación de la enseñanza, ya que es el propio profesorado el que asume el proceso de recogida de la información sobre sus actuaciones docentes. La autora también considera que la evaluación es más un camino que una meta dado que su principal objetivo es la mejora continua basada en el autoconocimiento de los puntos fuertes y débiles.

Si bien el principal objetivo de la CD es contribuir a la mejora continua, también se utiliza para procesos de promoción, acreditación o concursos — como en el caso de la Staff Educational Development—, para presentar la metodología de enseñanza-aprendizaje al alumnado; para proyectar una imagen positiva de la institución.

El contenido de la CD consiste básicamente en una descripción y análisis de las actuaciones que ha desarrollado el o la docente, los resultados que ha conseguido y qué va a hacer para mejorar dichos resultados. Además, incluye muestras de la calidad de la acción docente.

Se trata de un documento personal narrado en primera persona del singular, seguramente debido a su influencia anglosajona.

El o la docente debe describir los esfuerzos que se han hecho para impartir docencia de calidad y los resultados obtenidos. Es importante incluir todo tipo de resultados, tanto positivos como negativos, ya que lo importante es evidenciar que ha habido una autocrítica de la propia actuación, y, como consecuencia de la autocrítica, proponer un plan de mejora. 
Los materiales seleccionados como muestras evidencian el estilo del profesor. Las muestras sirven asimismo para recoger datos, analizarlos, interpretarlos y diseñar un cambio.

Debe ser breve y sintética. Se recomienda una extensión de entre 12 y 20 páginas, a las que se pueden ańadir los apéndices necesarios, es decir todas las muestras que se desee.

Visto que la actividad docente es muy diversa - incluye diseño de material de aprendizaje, proceso de enseñanza, evaluación del aprendizaje, evaluación de la enseñanza, participación en comisiones docentes, etc.la carpeta puede contribuir a organizar toda la documentación sobre el proceso pedagógico de forma integral y a documentar un proceso.

En función del uso que se la quiera dar se tendrá que decidir qué estructura debe tener.

Su objetivo puede ser sumativo, es decir, puede servir para evaluar al profesorado, o simplemente formativo, para la propia mejora profesional.

Hacerla por primera vez es un proceso complejo que requiere mucha dedicación, por ello se recomienda que haya acompañamiento.

La carpeta es un documento dinámico que se debería revisar periódicamente.

\section{Contenido de la carpeta docente}

La estructura de la CP puede variar en función de cuál sea su finalidad: autoevaluación, acreditación o evaluación por parte de la institución. La estructura que proponemos en este artículo es la usada en el programa de Formación Docente en Educación Superior de la Universitat Autònoma de Barcelona. Los apartados propuestos son: datos biográficos, filosofía docente, muestras y plan de mejora.

\section{Datos biográficos}

Este apartado consiste en la presentación del perfil del docente y su trayectoria académica y profesional. Puede incluir los siguientes aspectos: 
- Los estudios realizados, y la relación que tienen con las asignaturas que se imparten.

- La experiencia profesional vinculada a la materia.

- Las asignaturas impartidas a lo largo de la trayectoria académica, o en el período evaluado, indicando el número de créditos y horas impartidos, si se trata de asignaturas troncales u optativas, si se es la persona responsable, el número de estudiantes, tamaño de los grupos, perfil del alumnado. Se puede destacar, si fuera el caso, la versatilidad que se tiene si se han impartido asignaturas de ámbitos diversos o el perfil del alumnado de cada asignatura. Además, hay que indicar si se ha encargado del diseño de materiales.

- Los cargos académicos ocupados y tareas de coordinación académica.

- Coordinación o participación en comisiones de docencia y las funciones desempeñadas, así como si se ha participado en comisiones académicas, como comisiones de elaboración o revisión de plan de estudios, comisiones de evaluación de una titulación.

- La participación en proyectos de innovación docente.

- Cursos y seminarios recibidos o impartidos que repercutan en la mejora docente del profesorado.

- Las publicaciones (libros, capítulos o artículos) y las comunicaciones en congresos sobre docencia.

- La dirección de trabajos dirigidos: tesis, trabajos de fin de máster o trabajos de fin de grado.

- El número de quinquenios docentes concedidos.

- Participación en actividades de transición de secundaria a la universidad o de la universidad al mercado laboral.

- Participación en actividades para captar estudiantes: Jornadas de puertas abiertas, charlas en ayuntamientos, Salón de la Enseñanza, etc.

- Tutorización de alumnado con necesidades educativas especiales.

- Premios y distinciones docentes.

- Docencia en otras instituciones, tanto por invitación como por medio de una beca de movilidad docente.

\section{Filosofía docente}


La filosofía docente consiste en la concepción que tiene el profesorado de la enseńanza-aprendizaje.

Los aspectos que se pueden tratar en este apartado son:

- Los principios y valores sobre la educación superior que condicionan la manera de enseńar y cómo se trasladan a la actividad docente. El modelo de enseñanza-aprendizaje que se emplea en las aulas, haciendo hincapié en los valores y creencias que se tiene sobre la enseñanza y cómo se trasladan esas creencias a la práctica. Por ejemplo, se puede indicar si se utiliza un modelo constructivista, centrado en el papel activo del alumnado.

- Las metodologías de enseñanza que se utilizan, poniendo un énfasis especial en las metodologías activas que promueven el aprendizaje significativo, el aprendizaje basado en problemas, el enfoque por proyectos, el estudio de casos, el aprendizaje cooperativo, el aprendizaje experimental, etc. También se puede indicar el tipo de actividades participativas que se proponen.

- El uso que se hace de plataformas digitales, tanto para el desarrollo de las actividades de aprendizaje como para la comunicación, que ofrecen mayor flexibilidad a la situación personal del alumnado y porque al mismo tiempo fomentan la adquisición de la competencia digital.

- La creación de un entorno de aprendizaje inclusivo mediante el uso del diseño universal de aprendizaje, o de metodologías y actividades accesibles.

- El desarrollo de competencias transversales mediante el fomento del trabajo en equipo, el análisis crítico, el trabajo por proyectos, por indagación, etc.

- La actitud del docente en clase. Es decir, si se tiene conciencia en la necesidad de que las explicaciones sean claras y sigan una estructura lógica, si se pone suficiente entusiasmo para motivar al alumnado, la habilidad para plantear y responder preguntas...

- La enseñanza fuera del aula, como actividades de tutoría, la planificación y la coordinación de asignaturas, el asesoramiento de colegas.

- La participación del alumnado en el proceso. Se puede describir si el alumnado tiene poder de decisión sobre el calendario, las activi- 
dades programadas y la evaluación, y de qué manera se recoge su opinión.

- La evaluación. Es importante indicar si la evaluación es objetiva, qué criterios se usan, si se dispone de matrices de corrección (y si éstas están a disposición del alumnado antes de la realización de actividades), y el tipo de retroalimentación que reciben.

- El vinculo universidad-mercado laboral, esto es, si se simulan casos reales que el alumnado debe resolver, si se traen profesionales para dar a conocer la dinámica del mercado de trabajo, etc.

- La evolución del docente a través de la formación permanente (cursos y talleres tanto de innovación docente como del área de conocimiento).

\section{Muestras de efectividad}

Si el apartado anterior incluye una descripción de la concepción que tiene el o la docente sobre cómo debe ser la enseńanza para promover el aprendizaje, en este apartado se deben incluir muestras que corroboren de qué manera se aplica la filosofía docente. Este apartado supone un gran ejercicio de reflexión, ya que se debe seleccionar un número limitado de muestras y justificarlas.

Algunos de los documentos que se pueden adjuntar son:

- La programación de asignaturas. Esto puede mostrar si está contemplada la enseńanza basada en competencias o si se impulsa un enfoque concreto a nivel institucional.

- Cómo se integra la materia impartida en el área de conocimiento.

- Coordinación con el profesorado de otras asignaturas.

- Proceso de preparación de la asignatura y justificación del material utilizado, tanto el material docente como las unidades didácticas o las actividades que se ponen a disposición de los alumnos. Estos materiales pueden poner de manifiesto si se facilita la adquisición de competencias mediante actividades prácticas, el tipo de herramientas que deben usar, si se aplican los principios del diseño universal de aprendizaje para promover la igualdad de oportunidades, etc. 
- Interacción con el alumnado en el aula y fuera del aula, y cómo participa éste en el aprendizaje.

- Actividades que muestren la eficacia de la docencia:

- Cómo se aprovechan las TIC para enriquecer la enseñanza, mejorar las experiencias de aprendizaje, apoyar el aprendizaje personalizado y facilitar el acceso del alumnado con NEE.

- Cómo se explican conceptos o procesos difíciles, ejemplos que se dan o actividades que se han diseńado para ello.

- De qué manera se vincula la docencia con el mercado: explicaciones, actividades de simulación, metodologías de trabajo, visitas a empresas, etc.

- Estructura de las sesiones, es decir, cómo se articula la teoría con la práctica para promover la adquisición de competencias, y de qué manera se consigue mantener el interés del alumnado.

- Ejemplos de actividades de enseńanza-aprendizaje, tareas de evaluación, resultados de aprendizaje que demuestren que se sigue la filosofía docente mencionada en el apartado previo.

- Qué se hace para evaluar la propia enseñanza, si se utiliza algún documento para recoger los comentarios del alumnado (cuestionarios de la institución o cuestionarios diseñados ad-hoc, diarios, observación, etc.), la periodicidad con que se usan, de qué manera se revisa la práctica docente tras los resultados obtenidos.

- Qué se hace para mejorar el aprendizaje de los estudiantes: si se retoman ciertas tareas, se hacen tareas de refuerzo, se se diseñan tareas diversas para que puedan contextualizar y consolidar el aprendizaje, etc.

- Cómo se articula la evaluación del aprendizaje:

Justificación de la elección de las actividades de evaluación; por ejemplo: diseńo de tareas de evaluación diversas, no solo para evaluar distintas competencias, sino para promover distintos estilos de aprendizaje.

- Se evalúan las competencias o solo contenidos.

- Las tareas de evaluación se corresponden con los contenidos de aprendizaje trabajados y el nivel exigencia es equiparable al énfasis que se le ha puesto durante el curso. 
- Los estudiantes han participado en la definición de criterios de corrección.

- Las matrices de corrección de tareas, indicando si el alumnado disponía de ellas antes de la realización de la actividad.

- Instrumentos de evaluación diversos: cuestionarios, diarios, tareas, carpeta de aprendizaje, exámenes, etc.

- Cómo se articulan los diferentes tipos de evaluación: sumativa, diagnóstica, formativa, qué información se recoge en cada caso, qué acciones se toman en función de los resultados, el tipo de retroalimentación que se da, etc.

- Los agentes implicados en la evaluación, en este sentido, es importante indicar si se utilizan la autoevaluación, para que el propio alumno/a tome conciencia de su nivel de desempeño, y la coevaluación, o sea, si los propios compañeros/as tienen que valorar las tareas, y, en caso afirmativo, si disponen de criterios de evaluación, si eso sucede en la evaluación formativa o también en la sumativa, etc. - Utilidad de las actividades de evaluación.

- Resultados académicos y grado de aprovechamiento del alumnado, indicando si hay dificultades en la adquisición de ciertas competencias, causas de fracaso, etc.

Grado de satisfacción del alumnado con la actuación docente.

- Dificultades encontradas en la tarea docente.

- Sugerencias de compañeros y de los propios estudiantes. En este sentido, se pueden incluir los cuestionarios institucionales de la satisfacción del alumnado o correos electrónicos con comentarios de otros/as docentes o de alumnado.

- Formación docente recibida, y cómo se incorporado en el aula lo que se ha aprendido.

- Participación en comisiones docentes para la modernización del sistema educativo.

- Elaboración de materiales docentes, tanto libros de texto como materiales disponibilizados en plataformas de enseńanza-aprendizaje cerradas, páginas web propias, etc.

- Artículos publicados y comunicaciones en congresos relacionados con la innovación docente. 


\section{Plan de mejora}

A partir de las muestras aportadas en el apartado anterior, el profesorado debe extraer conclusiones de qué aspectos puede mejorar. Por ello, la carpeta docente debe incluir una planificación de los objetivos a corto, medio y largo plazo que se desean alcanzar. Los aspectos susceptibles de mejora son:

- Docencia. Aspectos susceptibles de mejora son la adaptación de asignaturas a un modelo basado en competencias, la revisión de los contenidos de aprendizaje, la introducción de más actividades prácticas, el diseño de descriptores del aprendizaje, la revisión de las tareas de evaluación, el diseño de rúbricas de evaluación, etc.

- Formación docente. Como en todas las profesiones, las metodologías docentes evolucionan y el profesorado debería reciclarse regularmente. Numerosas universidades disponen de un programa para el aprendizaje permanente de su plantilla, con cursos que van desde la evaluación por competencias, la enseńanza para grupos grandes, uso de ciertas tecnologías en el aula, el diseño universal de aprendizaje, la enseńanza con perspectiva de género, etc.

- Proyectos de innovación docente. Existen algunas convocatorias de proyectos de innovación docente. La ventaja de este tipo de proyectos es que a menudo los equipos de trabajo son multidisciplinares y se establecen redes de cooperación, de manera que todos se enriquecen de puntos de vista distintos.

- Participación en congresos. Existen congresos consagrados a la innovación docente y que son un excelente espacio no solo para presentar los resultados obtenidos de las diferentes experiencias docentes, sino también para construir una redes de cooperación, nacionales o internacionales, con los que crear sinergias.

\section{Apéndices}

Aquí se incluyen los elementos que muestran lo que se haya indicado en el apartado anterior. Los apéndices no tienen límite de extensión, pero, 
como sugiere Fernández March (2004), deben ser manejables para que otras personas lo puedan leer.

\section{Conclusiones}

La carpeta docente, como se ha podido ver, es un documento reflexivo sobre la calidad de la propia acción formativa. En un contexto en el que se aboga por la enseñanza basada en competencias, la CD parece ser un instrumento idóneo para presentar el desempeño a través de muestras de eficacia muy diversas y procedentes de diferentes agentes (el propio docente, el alumnado, la institución, compañeros, etc.).

Algunas de las ventajas del uso de la carpeta son:

- La reflexión sobre la calidad docente.

- La recopilación sistemática de datos que muestren la efectividad docente.

- La reflexión sobre la propia actividad.

- El diseño de un plan de mejora concreto, que permite introducir cambios paulatinos en la enseñanza.

- Al tratarse de un documento dinámico, que se debe revisar periódicamente, permite que la persona se autorregule y vaya enriqueciendo su actuación.

- Al ver la carpeta docente como un todo y tener que escoger ejemplos de su trabajo, se toma conciencia del propio desempeńo.

Sin embargo, también presenta algunos inconvenientes:

- Su diseño es un proceso complejo y largo, que se añade al resto de tareas del profesorado universitario.

- La mayoría de las agencias que evalúan la calidad del sistema universitario y las propias universidades, si bien fomentan su elaboración a través de cursos de formación permanente, no la consideran ni en los procesos de acreditación ni de contratación.

Tras considerar estas ventajas e inconvenientes, pensamos que el uso de la carpeta docente se debería fomentar en el entorno universitario. Las 
universidades y las agencias de evaluación deberían incluirla en los procesos de contratación y acreditación, ya que aporta datos no solo cuantitativos, sino también cualitativos.

El artículo da detalles de cómo se puede articular la carpeta docente, no obstante, sería recomendable averiguar qué porcentaje de profesorado universitario dispone de ella y qué usos reales le otorgan.

\section{Bibliografía}

BOB; CARMELA A. WHITE; DANIELA WONG GONZALEZ (2017). Metaanalysis of faculty's teaching effectiveness: Student evaluation of teaching ratings and student learning are not related. Studies in Educational Evaluation, v. 54: p. 22-42, 2017.

BORING, A.; OTTOBONI, K.; STARK, P. Student evaluations of teaching (mostly) do not measure teaching effectiveness". ScienceOpen Research, 2016. Doi: 10.14293/ S2199-1006.1.SOR-EDU.AETBZC.v1.

COLÉN, M. T.; GINÉ, N.; IMBERNON, F. La carpeta de aprendizaje del alumnado universitario: la autonomía del estudiante en el proceso de aprendizaje. Barcelona: Octaedro, 2006.

COMISIÓN EUROPEA. Comunicación de la Comisión al Parlamento Europeo, al Consejo, al comité Económico y Social Europeo y al Comité de las Regiones sobre una agenda renovada de la UE para la educación superior. COM/2017/0247 final, 2017. Disponible en: https://eur-lex.europa.eu/legal-content/ES/ TXT/?uri=CELEX:52017DC0247

COMISIÓN EUROPEA. Highl level Group on the Modernisation of Higher Education. Report to the European Commission on improving the quality of teaching and learning in Europe's higher education institutions, 2018. Disponible en: https://publications.europa. eu/en/publication-detail/-/publication/fbd4c2aa-aeb7-41ac-ab4c-a94feea9eb1f

DAVIES, A.; LEMATHIEU, P. Assessment for Learning: Reconsidering Portfolios and Research Evidence. In: Segers; M.; Dochy, F. (Ed.). Optimising New Modes of Assessment: In Search of Qualities and Standards. The Netherlands: Kluwer Academic Publishers, 2003, p. 141-169.

DRIESSEN, E., et al. The Use of Qualitative Research Criteria for Portfolio Assessment as an Alternative to Reliability Evaluation: A Case Study. Medical Education, v. 39, p. 214-220, 2005. Doi:10.1111/med.2005.39.issue-2.

EDGERTON, R. et al. The Teaching Portfolio. Capturing the Scholarship in Teaching. Washington: American Association for Higher Education, 1995. 
FENSTERMACHER, G. D.; RICHARDSON, V. On making determinations of quality in teaching. Teachers College Record, v. 107, v. 1, p. 186-213, 2005.

FERNÁNDEZ MARCH, A. El portafolio docente como estrategia formativa y de desarrollo profesional. Educar, v. 33, p. 127-142, 2004.

FUENTES, M.; GALÁN-MAÑAS, A.; OJEDA-SUÁREZ, M.E. El Portafolio Docente como instrumento de reflexión y mejora del profesorado universitario, 2011.

FUENTES, M.; GALÁN-MAÑAS, A.; OJEDA-SUÁREZ, M.E. La elaboración de la carpeta docente (portafolio docente). Dossier de actividades, 2011. http://ddd.uab.cat/ record/73619.

GALÁN-MAÑAS, A. La enseñanza de la traducción en la modalidad semipresencial. Tesis doctoral. Universitat Autònoma de Barcelona, 2009.

GALÁN-MAÑAS, A. Learning portfolio in translator training: the tool of choice for competence development and assessment, The Interpreter and Translator Trainer, 2016. Doi: 10.1080/1750399X.2015.1103108

GALÁN-MAÑAS, A. Professional portfolio in translator training: professional competence development and assessment. The Interpreter and Translator Trainer, v. 13, n. 1, p. 44-63, 2018.Doi: 10.1080/1750399X.2018.1541295.

GALÁN-MAÑAS, A., FUENTES, M.; OJEDA-SUÁREZ, M.E. El portafolio docente. Metodología y claves para su elaboración. Managua: Ed. Hispamer, 2013.

GALÁN-MAÑAS, A.; Fuentes, M.; Ojeda-Suárez, M.E. La carpeta de aprendizaje del estudiante universitario. Diseño e implementación. Managua: Hispamer, 2013.

JENSEN, B. Complexity and Scale in Teaching Effectiveness Research: Reflections from the MET Study. Education policy analysis archives, v. 27, n. 7, p. 6-15, 2019.

LITTLE, D. The European Language Portfolio: Structure, Origins, Implementation and Challenges. Language Teaching, v. 35, n. 3, p. 182-189, 2002. Doi:10.1017/ S0261444802001805.

PAULSON, L., PAULSON, P.; Meyer, C. What Makes a Portfolio a Portfolio? Educational Leadership, v. 48, n. 60-63, 1991.

PRENDES, P.; Mar, S. El portafolio como herramienta de evaluación. In: Porto, M. (ed.) Técnicas de evaluación en el EEES. Murcia: Edit.um., 2010.

Recebido em 2 dez. 20I9/ Aprovado em I8 dez. 2019

\section{Para referenciar este texto:}

GALÁN-MAÑAS, A. La carpeta docente. Instrumento para la evaluación de la calidad docente. EccoS - Revista Científica, São Paulo, n. 51, eI6258, out./dez. 2019. Disponível em: https://doi.org/I0.5585/EccoS.n5I.I6I58. 